\section{$\mathbf{m} / \mathbf{S}$}

médecine/sciences $1995 ; 11: 517-8$

\title{
TOUT CE QUE VOUS AVEZ TOUJOURS VOULU SAVOIR SUR LE TESTICULE SANS JAMAIS OSER LE DEMANDER
}

\section{Bernard Jégou}

\section{ADRESSE ET TIRÉS À PART}

B. Jégou : directeur de recherche à l'Inserm, directeur du groupe d'étude de la reproduction chez le mâle (GERM), directeur de l'U. 435 de l'Inserm. Université de Rennes I, campus de Beaulieu, avenue du Général-Leclerc, 35042 Rennes, France.

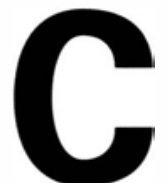

hacun sait évidemment la place que le testicule occupe, tant dans la reproduction des espèces, que dans l'imaginaire et dans la vie sociale des individus. Le déguisement de Woody Allen en spermatozoïde, dans le film qui a inspiré le titre de cet éditorial*, n'a fait que le rappeler avec beaucoup d'humour ; l'impact récent du film de $\mathrm{G}$. Corbiau sur la vie imaginée de Carlo Broschi, dit Farinelli, le plus célèbre castrat du $\mathrm{XVIII}^{\mathrm{e}}$ siècle, le rappelle à nouveau avec beaucoup d'émotion.

Le mot testis et son diminutif testiculi, dont est dérivé celui de testicule, est un mot latin qui signifie "témoin". Son utilisation par les Romains pour désigner les gonades mâles pourrait découler du fait que les testicules " témoignent " de la virilité de l'homme et/ou que, selon certains auteurs, dans plusieurs civilisations antiques, pour jurer ou témoigner, l'individu devait placer sa main sur ses testicules ou sur ceux d'un partenaire (au moins une scène de la Bible en attesterait !) [1].

Au-delà de l'histoire et des anecdotes les plus surprenantes, et même si le rôle des spermatozoïdes dans la fécondation n'a été vraiment établi par Prévost et Dumas qu'en 1824 [2], certains aspects essentiels de la fonction des testicules ont été très tôt perçus, du fait de leur particulière accessibilité. Dès le néolithique, les hommes pratiquaient la castration pour domestiquer et engraisser cer-

* Titre original $d u$ film : Everything you always wanted to know about sex but were afraid to ask. tains animaux et, au moins 2000 ans avant Jésus-Christ, certaines sociétés recouraient à la castration pour punir l'adultère [3]. Ce n'est donc très probablement pas un hasard si l'endocrinologie, en tant que telle, est née en 1849 des expériences de Berthold [4] démontrant que la transplantation du testicule est capable de maintenir les caractères sexuels mâles chez le coq castré et si le concept d'hormone doit tant aux expériences d'auto-injection d'extraits testiculaires de Brown-Sequard [5], dont Zola s'inspira pour écrire le dernier volume de la série des Rougon-Macquart, le Docteur Pascal. La dualité de la fonction du testicule production des spermatozoïdes et sécrétion des hormones mâles (androgènes) - est maintenant bien établie, de même qu'il est bien compris que ces deux fonctions sont indissociables. En effet, des niveaux adéquats d'androgènes sont indispensables, tant pour assurer une spermatogenèse normale que pour garantir la transmission des spermatozoïdes au sexe féminin, puisque celle-ci repose sur le développement correct des caractères sexuels secondaires et sur un certain comportement sexuel, ces deux aspects étant également dépendants des androgènes.

Etant donné l'ancienneté des observations et des approches expérimentales appliquées dans ce domaine, il peut sembler paradoxal qu'à la fin de ce siècle, les mécanismes du contrôle de la spermatogenèse soient si mal connus. Cette méconnaissance du fonctionnement précis du testicule explique que, dans l'espèce humai- 
ne, l'étiologie des stérilités masculines reste le plus souvent inconnue, alors que, dans presque un cas sur deux d'infécondité, c'est la fertilité de l'homme qui est en cause [6]. Elle explique aussi l'absence virtuelle de traitement des dysfonctionnements de la spermatogenèse, ainsi que les difficultés considérables de mise au point de contraceptifs masculins utilisables à grande échelle, autres que le préservatif. Que dire aussi du fait que nous en soyons réduits pour le moment à de vaines spéculations pour expliquer l'augmentation sensible du taux des cancers testiculaires et la baisse alarmante de la production spermatique chez l'homme sain, constatées au cours des dernières décennies $[7,8]$ ? Pour compléter ce sombre tableau, notons que le testicule est un des organes les plus vulnérables aux effets des xénobiotiques et des rayonnements, comme en témoignent les effets désastreux des thérapies anticancéreuses et des pesticides sur la spermatogenèse. Pourtant, la reproduction chez le mâle reste le domaine le moins exploré par les toxicologues... Enfin, bien que l'on connaisse le rôle crucial du sperme dans la transmission du SIDA et des hépatites, les voies d'entrée et les sites de réplication des virus dans le tractus génital mâle sont pratiquement inconnus.

Comment expliquer ce retard ? Les chercheurs et cliniciens travaillant dans le champ de l'andrologie (discipline qui couvre l'étude de tous les aspects de la physiologie, de la pathologie de la reproduction et de la sexualité chez le mâle) auraient-ils failli à leur mission ? Le laisser croire serait vraiment très injuste vis-à-vis des générations de morphologistes, de biochimistes, de biologistes et de cliniciens qui y ont consacré leur expertise et leurs efforts de recherche. En réalité, le testicule est devenu un organe "sexy " à étudier, en raison de la très grande diversité et de la spécificité de l'expression génétique des cellules testiculaires [9]. Des avancées et des convergences disciplinaires importantes ont lieu, comme en témoigne le contenu de ce numéro que médecine/sciences a généreusement ouvert aux cher-
Toutefois, l'analyse approfondie des causes fondamentales de ce retard est nécessaire afin que l'intérêt accru des chercheurs pour le testicule se traduise par des progrès fondamentaux et appliqués encore plus décisifs. Ces causes pourraient être de trois ordres principaux : (1) pour des raisons socio-culturelles, l'attention que la Société a portée et les moyens qu'elle a consacrés à ce domaine ont toujours été très insuffisants. La stérilité et la contraception, en particulier, ont été et sont probablement encore très (trop) souvent perçues comme des problèmes spécifiquement féminins. Songeons également au fait que, si grâce à l'action d'enseignants très motivés, quelques universités françaises ont créé des enseignements d'andrologie, en revanche, cette discipline ne figure toujours pas dans le cursus normal du futur médecin, contrairement à la gynécologie ; (2) pour des raisons économiques qu'il serait trop long d'exposer ici, dans le domaine de l'élevage, à l'exception des moyens consacrés à la mise au point des techniques de cryoconservation du sperme, les investissements les plus importants ont porté sur la femelle. Les retombées très positives qui en découlent en reproduction humaine s'appliquent donc essentiellement à la femme (voir, par exemple, différentes techniques utilisées pour l'assistance médicale à la procréation) ; (3) enfin, que ce soit chez l'homme ou chez l'animal, l'organisation spatio-temporelle de la spermatogenèse est extraordinairement sophistiquée et repose sur une structure anatomique de l'épithélium des tubules séminifères, où sont formés les spermatozoïdes, unique du point de vue de sa complexité [10]. Il est donc clair que l'exploration de la fonction testiculaire se heurte à des obstacles intrinsèques hors du commun.

Il est difficile au biologiste de savoir si le regard que porte la société sur la masculinité (par exemple, dans le domaine de la contraception) se modifie et peut conduire à un développement des recherches dans ce domaine. Toutefois, compte tenu de l'importance des problèmes de santé publique rapportés ci-dessus, il serait impensable que l'andrologie ne reçût pas plus d'attention et de soutien. La technique d'injection intracytoplasmique du spermatozoïde dans l'ovocyte humain bouleverse la procréation médicalement assistée et le traitement de la stérilité masculine en permettant à certains hommes jusqu'alors infertiles d'assurer leur descendance avec un seul gamète défectueux et, peut-être même, avec une cellule germinale immature! [11]. Il ne faudrait cependant pas que l'émergence de cette innovation technologique, qui n'est pas sans poser de très sérieux problèmes éthiques, crée la dangereuse illusion que la Société peut faire l'économie de développer l'étude approfondie des mécanismes du contrôle de la spermatogenèse, de la maturation du spermatozoïde et de la fécondation $\square$

\section{RÉFÉRENCES}

1. Bremner WJ. Historical aspects of the study of the testis. In: Burger $\mathrm{H}$, de Kretser $\mathrm{DM}$, eds. New York : Raven Press, 1981 : 1-8. 2. Prévost JL, Dumas JBA. Nouvelle théorie de la génération. Ann Sci Nat $1824 ; 2: 129$ 49 . 3. Setchell BP. Male reproduction. In: Setchell BP, ed. Benchmark papers in human phy siology, vol. 17. New York : Van Nostrand Reinhold Compagny Scientific and AcadeReinhold Compagny Scie
mic Editions, 1984: $1-406$.

4. Berthold AA. Transplantation der Hoden. Arch Anat Physiol Wiss Med 1849; $16: 4246$.

5. Brown-Séquard CE. Expérience démontrant la puissance dynamogénique chez l'homme d'un liquide extrait de testicules d'animaux. Arch Phys Norm Pathol 1889; 21 : 651.

6. Thonneau P, Patureau J, Moyse C, Marchand S, Tallec A, Ferial ML, Lansac J, Lopes $P$, Tabaste JM, Ducot B, Spira A. L'infécondité en France : résultats d'une étude multicentrique dans trois départements francais (1988-1989). Contracept Fertil Sex 1992 ; 1 : 2732.

7. Giwercman A, Skakkebaek NE. The human testis : an organ at risk ? Int J Androl $1992 ; 15: 373-5$.

8. Auger J, Kunstmann JM, Czyglik F, Jouannet $P$. Decline in semen quality among fertile men in Paris during the past 20 years. $N$ EnglJ Med 1995; 332 : 281-5.

9. Barouki R. Expression des gènes au cours de la spermatogenèse. médecine/sciences $1992 ; 8: 53240$.

10. Fawcett DW. Ultrastructure and function of the Sertoli cell. In: Hamilton DW, Greep RO, eds. Handbook of physiology, vol. V. Baltimore : Williams and Wilkins, $9975: 21-55$. 11. Vanderzwalmen P, Bertin G, Nijs M, Lejeuine B, Schoysman R. Fécondation ovocytaire par spermatide dans un programme de fécondation in vitro. Andrologie 1994; 4 : $523-4$. 